\title{
Role of a Checklist to Improve Patient Safety in Interventional Radiology
}

\author{
Soumil Singhal ${ }^{1, \odot} \quad$ Mangerira Chinnappa Uthappa ${ }^{1, \oplus}$ \\ ${ }^{1}$ Department of Intervention Radiology and Intervention Oncology, \\ BGS Gleneagles Global Hospital, Kengeri, Bangalore, Karnataka, India \\ Address for correspondence Soumil Singhal, MD Radiodiagnosis, \\ Department of Intervention Radiology and Intervention Oncology, \\ BGS Gleneagles Global Hospital, Kengeri, Bangalore, 560060, \\ Karnataka, India (e-mail: drsoumilsinghal75@gmail.com).
}

\begin{abstract}
A checklist can be defined as a comprehensive formal list of essential actions to be taken in a specific fashion. This concept has been extended from the aviation industry to health care to improve patient outcome and patient satisfaction with a significant reduction in complication rates. This review article aims to assess the importance and benefits associated with the use of a well-formulated checklist while performing the various minimally invasive image-guided procedures. Various databases including PubMed, Medline, Scopus, and Cochrane were searched for using various keywords

Keywords

- interventional radiology

- patient safety

- vascular interventions including "Checklist," "Radiology," "Interventional Radiology," "Image-Guided Procedure," and "minimally invasive procedure." The use of a checklist is the way ahead especially when patients today require minimal risk but demand high-quality care. Implementation of such an easy-to-perform tailor-made mechanism can significantly improve patient outcome and patient satisfaction.
\end{abstract}

\section{Introduction}

The concept of a checklist has existed for several years. A checklist is defined as a comprehensive formal list of essential actions to be taken in a specific fashion. This concept has been used in several highly systematic and risk-based industries such as the aviation industry where the use of checklist has demonstrated several advantages. This concept has now been extended to the health care to improve patient outcome and patient satisfaction with a significant reduction in complication rates. A checklist functions as a quality assurance memory-based tool in the form of a list of things to do by merely categorizing and subcategorizing them. ${ }^{1}$ A malpractice is an adverse event that occurs after a duty of care is established between a patient and a physician. ${ }^{2}$ Whang et al in their study reported that procedure-related complications were the second most common cause of malpractice claims against radiologists. Vascular injury was most common among all the complications in this study. ${ }^{3}$ They also noted that an inadequate, incomplete communication with the patient as the third most common cause for malpractice claims. ${ }^{3}$ Malpractice lawsuits are an unfortunate consequence of complication that can take years to resolve. These can be avoided by various methods of which use of a pre-procedure checklist is a very easy-to-perform and very effective mean to keep oneself safe from such ordeal. This review article aims to assess the importance and benefits associated with the use of a well-formulated checklist while performing the various minimally invasive image-guided procedures.

\section{History of Checklists}

A checklist is a list of relevant actions or important tasks that are to be performed in a specific order or succession. A book by Gawande titled The Checklist Manifesto: How to Get Things Right states how complex tasks have become and the need of assistance to deal with this complexity especially in the background of the vast array of knowledge and numerous techniques available these days. ${ }^{4}$ Checklists have been previously used in several high-risk industries such as aviation industry and power industry to aid in the smooth running of received

December 27, 2018

accepted after revision

February 18, 2019

published online

July 15, 2019
DOI https://doi.org/

$10.1055 / \mathrm{s}-0039-1693536$

ISSN 2457-0214.
(C)2019 by Indian Society of

Vascular and Interventional

Radiology
License terms

()(1) $\odot \circledast$ 
operations and safety of the people involved. In the aviation industry, the use of checklist dates back to the time before World War II. The use of a checklist in health care is ideal as checklists help improve both patient management and their safety. In 2003, The Joint Commission designed The Universal Protocol, which was created to prevent wrong person, wrong procedure, and wrong site surgery. It consisted of the parts including (a) pre-procedure verification, (b) marking the procedure site, and (c) timeout immediately before the procedure. $^{5}$ The World Health Organization (WHO) in 2009 published a 19-point surgical care checklist with an aim to reduce surgery-related morbidity and mortality. ${ }^{6}$ Haynes et al found that implementation of this reduced both surgical complication rate from 11 to $7 \%$ and morbidity rate from 1.5 to $0.8 \% .^{7}$ However, the WHO checklist had few limitations including surgical Hawthorne effect and failure to control confounding factors. SURgical Patient Safety System (SURPASS) checklist that is a much more comprehensive list with even better outcomes concerning morbidity and mortality. ${ }^{8}$ A systematic review article reported that 1 in 10 patients during their hospital stay developed adverse effect, of whom one-half were secondary to an invasive procedure. ${ }^{8}$ de Vries et $\mathrm{al}^{9}$ evaluated the importance of the use of checklist by performing a study with the use of a 100-item checklist made by use of about 11 different checklists dealing with various aspect of patient care. It included pre-/intra-/postoperative care and orders; imaging; printed information; right site/side verification; and discharge instructions that were completed by nurses, ancillary staff, surgeons, and anesthetists. The study reported reduction in complication rate (15.4-10.6\%) and mortality rate $(1.5-0.8 \%)^{9}{ }^{9}$

\section{Various Checklists in Interventional Radiology}

Interventional radiology is a highly dynamic and evolving field in terms of technical and instrumental advancement sharing several features with surgery. A checklist in intervention radiology would be as useful as it was in surgery. Various checklists have been proposed in intervention radiology. Based on the SURPASS checklist, Koetser et $\mathrm{al}^{10}$ published a 27-point checklist for an image-guided procedure called a RADiological Patient Safety System (RADPASS) checklist. The RADPASS checklist was created after reviewing all the standards and guidelines along with all the various adverse effect and complications associated with various interventional procedures. ${ }^{11-21}$ The RADPASS checklist has two parts: Part A (Planning and Preparation) and Part B (Procedure). Part B was further divided into B1 (just before starting) and B2 (post procedure). Part A comprises the following questions: (a) requisition form, (b) prior history, (c) relevant imaging, (d) indication, (e) preanesthetic evaluation (for general anesthesia), (f) contraindication (if any), (g) contrast allergy checked, (h) hospital admission, (i) lab and coagulation profile, and (j) medication and equipment related to the procedure. Part B1 comprises (a) the presence of intravenous (IV) line, (b) procedure explained to both patient and family, (c) possible complications explained to the patient (family), (d) right site, (e) right patient, (f) right procedure, (g) right side, and (h) antibiotic administration. Part B2 portion of the list comprises (a) post-procedure notes and orders, (b) procedure images sent to picture archiving system, (c) biopsy sample checked/ labeled/dispatched, (d) procedure and results explained to the patient/parents/referring physician, (e) report, (f) billing, and (g) follow-up appointment given. All parts were assessed either by an interventional radiologist (IR)/fellow/resident, under the supervision of a senior IR. Implementation of the checklist helped in the complete reduction in postponement rate $(10-0 \%)$ and also in the decrease in the process deviation rate to $5 \%$. The checklist was reported to be user friendly and, at the same time, increase patient efficiency and safety awareness. However, this checklist had a few limitations as suggested by the author. First, the effect of the checklist was evaluated in a before-after setting that might have been biased based on the case and the time frame. Second, the author suggests that the findings of their checklist might have been affected by the Hawthorne effect as an individual modifies his/her actions in response to being observed. Third, the impact of the checklist was only evaluated in a small group of patients. ${ }^{10}$

Lee et $\mathrm{al}^{22}$ based on the WHO checklist and RADPASS checklist, proposed the CIRSE IR checklist. This checklist has three sections: (a) Procedure planning, (b) "Sign-in," and (c) "Sign-out." Procedure planning portion is checked by the IR/ward nurse and comprises the following: (a) case discussed with referring physician, (b) prior imaging reviewed, (c) if relevant medical history is taken, (d) if informed consent was taken, (e) if contrast-induced nephropathy prophylaxis is given, (f) if all the specific tools are present/ordered, (g) if fasting orders are given, (h) if relevant lab tests are ordered, (i) if anesthesiologist is needed, ( $\mathrm{j}$ ) if anticoagulant medication is stopped, ( $\mathrm{k}$ ) if post-interventional, intensive care unit (ICU) bed is required, and (l) contrast allergy prophylaxis if necessary. During "Sign-in," the following points are checked by the resident/nurse/staff/IR: (a) if all team members are introduced, (b) if all records are with patient, (c) correct patient/ side/site, (d) if patient is fasting, (e) is IV access is present, (f) if monitoring equipment is attached, (g) if the coagulation screen/lab tests are checked, (h) if allergies and/or prophylaxis are checked, (i) if antibiotics/other drugs are administered, and (j) if the consent/complications are discussed with the patient/family. During "Sign-out," the following points are checked by the IR: (a) if postoperative note is written, (b) if vital signs are normal during the procedure, (c) if all the medication given are recorded, (d) if lab tests are ordered, (e) if all samples are labeled and sent to lab, (f) if procedure results are discussed with patient and referring physician, $(\mathrm{g})$ if post-discharge instruction are given, (h) if follow-up tests/ imaging are ordered, and (i) if follow-up OPD appointments are made. The checklist aimed at improving patient safety in Europe and is readily available to download in various European languages on the CIRSE website. Corso et $\mathrm{al}^{23}$ proposed a 20-item timeout checklist based on the CIRSE checklist. The checklist reduced all adverse effect in the first year and also raised awareness of patient safety among various health care teams. ${ }^{24-27}$ 
Rafiei et al reported another checklist to improve patient safety in the Society of Interventional Radiology Safety and Health Committee. ${ }^{28}$ The checklist was created as several previously reported checklists did not demonstrate satisfactory improvement is patient safety. ${ }^{29,30}$ They suggested that the drawbacks from different checklists were the cause for failure prompting a much more comprehensive checklist based on the local team's operational requirements and their case mix. The checklist consists of (a) at least two patient identifiers (patient name, patient date of birth, patient medical record number), (b) procedure details, (c) procedure site mark, (d) allergies, (e) contrast agent, (f) antibiotics administration time, (g) sedation plan, (h) laboratory tests (international normalized ratio [INR], platelets, partial thromboplastin time [PTT], creatinine), (i) blood products (type and screen), (j) prior imaging, ( $\mathrm{k}$ ) specimen collection, (l) radiation safety (staff protective and monitoring equipment, optimize low-dose radiation, patient protection), (m) magnetic resonance imaging (MRI) safety (magnetic resonance-compatible equipment and instruments, patient and staff screening), (n) need for special equipment, (o) additional safety concern, (p) consent confirmation, (q) patient radiation history, $(r)$ pregnancy test result, (s) procedure sterility classification, and (t) prophylactic allergy premedication confirmation.

\section{Creating a Checklist}

A checklist is a tool that reduces reliance on memory and establishes a mechanism to check and recheck elements that could be missed due to human tendencies. When used correctly, it can improve patient outcome. Verdaasdonk et al suggest that when the checklist is small with few items, it can miss critical components and can negatively affect by complicating the process when it is complex and time

Table 1 A tailor-made checklist for interventional radiology procedures according to our experience

\begin{tabular}{|c|c|c|c|}
\hline \multicolumn{4}{|l|}{$\begin{array}{l}\text { Name: ......... } \\
\text { Diagnosis: }\end{array}$} \\
\hline \multirow[t]{12}{*}{ Pre-procedure } & Parameters & Yes & No \\
\hline & History reviewed & & \\
\hline & Allergies, including contrast allergies & & \\
\hline & Imaging studies reviewed & & \\
\hline & Procedure explained & & \\
\hline & Informed consent & & \\
\hline & Preanesthetic evaluation & & \\
\hline & Indication for procedure & & \\
\hline & Coagulation profile & & \\
\hline & Procedure-related equipment/material/medication & & \\
\hline & Relevant lab evaluation & & \\
\hline & Overnight fasting & & \\
\hline \multirow[t]{6}{*}{ Procedure } & Intravenous (IV) access & & \\
\hline & Consent & & \\
\hline & Clearance & & \\
\hline & Confirm fasting & & \\
\hline & Right side/right patient/right procedure & & \\
\hline & Antibiotic administered & & \\
\hline \multirow[t]{5}{*}{ Post-procedure } & Post-procedure notes and instructions & & \\
\hline & Sample labeled and sent & & \\
\hline & $\begin{array}{l}\text { Procedure and outcome discussed with patients' } \\
\text { relatives }\end{array}$ & & \\
\hline & $\begin{array}{l}\text { Procedure and outcome discussed with } \\
\text { referring physician }\end{array}$ & & \\
\hline & Post-discharge instruction with follow-up instruction & & \\
\hline
\end{tabular}


consuming. ${ }^{31}$ When not implemented well, it can be noneffective. ${ }^{32} \mathrm{~A}$ checklist needs to be tailor-made according to one's institutional requirement keeping it simple yet entirely effective in improving the patients care. Its implementation can be possible by developing standardized protocols, educating staff, and promoting a safety culture ${ }^{33}$ within the hospital ecosystem. The digitization of this system is the way ahead as it provides easy review and better operation. Digital checklists have several advantages: it helps in streamlining the processes and offers a pool of previous patient data allowing easier accessibility and reducing paper-based checklist errors. ${ }^{34}$ Gottumukkala et al found a significant improvement in teams performing pediatric interventions when timeout was combined with the audiovisual recording system and continual feedback over 3 years. ${ }^{35}$

A checklist we consider apt for our institution ( - Table $\mathbf{1}$ ) includes three sections that are evaluated from the time of admission to the discharge of any patient, for an imageguided procedure. It consists of three components: (a) preprocedure (history reviewed, allergies, including contrast allergies, imaging studies evaluated, procedure explained, informed consent, preanesthetic evaluation, indication for procedure, coagulation profile, procedure-related equipment/material/medication, relevant lab evaluation, and overnight fasting), (b) procedure (IV access, consent, clearance, confirm fasting, right side/right patient/right procedure, and antibiotic administered), and (c) post-procedure (post-procedure notes and instructions, sample labeled and sent, procedure and outcome discussed with patients relatives, procedure and outcome explained with referring physician, and post-discharge instruction with follow-up instruction). All components, as suggested in this checklist, are performed regularly by the support of our memory; however, we recommend accomplishing the same in the form of a paper checklist or digital form in all patients for better documentation and to avoid missing any component during times of hurry.

\section{Conclusion}

The use of a checklist is the way ahead especially when patients today require minimal risk but demand high-quality care. Implementation of such an easy-to-perform tailor-made mechanism can significantly improve patient outcome and patient satisfaction.

\section{Conflicts of Interest}

None.

\section{References}

1 Morrow DG, Leirer VO, Andrassy JM, Hier CM, Menard WE. The influence of list format and category headers on age differences in understanding medication instructions. Exp Aging Res 1998;24(3):231-256

2 Carrafiello G, Floridi C, Pellegrino C, Nocchi Cardim L, Ierardi AM, Fugazzola C. Errors and malpractice in interventional radiology. Semin Ultrasound CT MR 2012;33(4):371-375
3 Whang JS, Baker SR, Patel R, Luk L, Castro A III. The causes of medical malpractice suits against radiologists in the United States. Radiology 2013;266(2):548-554

4 Gawande A. The Checklist Manifesto: How to Get Things Right. New York, NY: Metropolitan Books 2002

5 The Joint Commission. National patient safety goals. The Joint Commission website. Available at: www.jointcommission. org/assets/1/6/2015_NPSG_HAP.pdf. Accessed November 22, 2018

6 World Health Organization (WHO). WHO guidelines for safe surgery. WHO website. Available at: whqlibdoc.who.int/ publications/2009/9789241598552_eng.pdf. Accessed November 22,2018

7 Haynes AB, Weiser TG, Berry WR, et al; Safe Surgery Saves Lives Study Group. A surgical safety checklist to reduce morbidity and mortality in a global population. N Engl J Med 2009;360(5):491-499

8 de Vries EN, Prins HA, Crolla RMPH, et al; SURPASS Collaborative Group. Effect of a comprehensive surgical safety system on patient outcomes. N Engl J Med 2010;363(20):1928-1937

9 de Vries EN, Hollmann MW, Smorenburg SM, Gouma DJ, Boermeester MA. Development and validation of the SURgical PAtient Safety System (SURPASS) checklist. Qual Saf Health Care 2009;18(2):121-126

10 Koetser IC, de Vries EN, van Delden OM, Smorenburg SM, Boermeester MA, van Lienden KP. A checklist to improve patient safety in interventional radiology. Cardiovasc Intervent Radiol 2013;36(2):312-319

11 Angle JF, Nemcek AA Jr, Cohen AM, et al; SIR Standards Division; Joint Commission Universal Protocol for Preventing Wrong Site, Wrong Procedure, Wrong Person Surgery. Quality improvement guidelines for preventing wrong site, wrong procedure, and wrong person errors: application of the joint commission "Universal Protocol for Preventing Wrong Site, Wrong Procedure, Wrong Person Surgery" to the practice of interventional radiology. J Vasc Interv Radiol 2008;19(8):1145-1151

12 Duncan JR. Strategies for improving safety and quality in interventional radiology. J Vasc Interv Radiol 2008;19(1):3-7

13 Jacobs B, Duncan JR. Improving quality and patient safety by minimizing unnecessary variation. J Vasc Interv Radiol 2009;20(2):157-163

14 Miller DL. Safety in interventional radiology. J Vasc Interv Radiol 2007;18(1 Pt 1):1-3

15 Stecker MS. Root-cause analysis. J Vasc Interv Radiol 2007; 18(1 Pt 1 ): 5-8

16 Borgstede JP, Zinninger MD. Radiology and patient safety. Acad Radiol 2004;11(3):322-332

17 Cardella JF, Kundu S, Miller DL, Millward SF, Sacks D; Society of Interventional Radiology. Society of Interventional Radiology clinical practice guidelines. J Vasc Interv Radiol 2009; 20(7 Suppl):S189-S191

18 Knight F, Galvin R, Davoren M, et al. The evolution of universal protocol in interventional radiology. J Radiol Nurs 2006;25:106-115

19 Sridhar S, Duncan JR. Strategies for choosing process improvement projects. J Vasc Interv Radiol 2008;19(4):471-477

20 Thrall JH. Quality and safety revolution in health care. Radiology 2004;233(1):3-6

21 Tuong B, Shnitzer Z, Pehora C, et al. The experience of conducting mortality and morbidity reviews in a pediatric interventional radiology service: a retrospective study. J Vasc Interv Radiol 2009;20(1):77-86

22 Lee MJ, Fanelli F, Haage P, Hausegger K, Van Lienden KP. Patient safety in interventional radiology: a CIRSE IR checklist. Cardiovasc Intervent Radiol 2012;35(2):244-246 
23 Corso R, Vacirca F, Patelli C, Leni D. Use of "Time-Out" checklist in interventional radiology procedures as a tool to enhance patient safety. Radiol Med (Torino) 2014;119(11):828-834

24 Pronovost P, Needham D, Berenholtz S, et al. An intervention to decrease catheter-related bloodstream infections in the ICU. N Engl J Med 2006;355(26):2725-2732

25 Walsh TS, Dodds S, McArdle F. Evaluation of simple criteria to predict successful weaning from mechanical ventilation in intensive care patients. Br J Anaesth 2004;92(6):793-799

26 Wolff AM, Taylor SA, McCabe JF. Using checklists and reminders in clinical pathways to improve hospital inpatient care. Med J Aust 2004;181(8):428-431

27 Mayo PH, Hegde A, Eisen LA, Kory P, Doelken P. A program to improve the quality of emergency endotracheal intubation. J Intensive Care Med 2011;26(1):50-56

28 Rafiei P, Walser EM, Duncan JR, et al; Society of Interventional Radiology Health and Safety Committee. Society of Interventional Radiology IR pre-procedure patient safety checklist by the Safety and Health Committee. J Vasc Interv Radiol 2016;27(5):695-699

29 Urbach DR, Govindarajan A, Saskin R, Wilton AS, Baxter NN. Introduction of surgical safety checklists in Ontario, Canada. N Engl J Med 2014;370(11):1029-1038
30 Sewell M, Adebibe M, Jayakumar P, et al. Use of the WHO surgical safety checklist in trauma and orthopaedic patients. Int Orthop 2011;35(6):897-901

31 Verdaasdonk EG, Stassen LPS, Widhiasmara PP, Dankelman J. Requirements for the design and implementation of checklists for surgical processes. Surg Endosc 2009;23(4):715-726

32 Reames BN, Krell RW, Campbell DA Jr, Dimick JB. A checklist-based intervention to improve surgical outcomes in Michigan: evaluation of the Keystone Surgery program. JAMA Surg 2015;150(3):208-215

33 Conley DM, Singer SJ, Edmondson L, Berry WR, Gawande AA. Effective surgical safety checklist implementation. J Am Coll Surg 2011;212(5):873-879

34 Boorman D. Today's electronic checklists reduce likelihood of crew errors and help prevent mishaps. ICAOJ 2001;56(1): $17-21$

35 Gottumukkala R, Street M, Fitzpatrick M, Tatineny P, Duncan JR. Improving team performance during the preprocedure time-out in pediatric interventional radiology. Jt Comm J Qual Patient Saf 2012;38(9):387-394 